\title{
Individual differences in pulse brightness perception
}

\author{
RICHARD W. BOWEN \\ Loyola University of Chicago, Chicago, Illinois 60626 \\ ROBERT SEKULER and CYNTHIA J. OWSLEY \\ Northwestern University, Evanston, Illinois 60201 \\ and \\ KATHRYN A. MARKELL \\ Loyola University of Chicago, Chicago, Illinois 60626
}

\begin{abstract}
When brightness-pulse duration relations are studied with a simultaneous brightness discrimination procedure, three classes of observers emerge (Bowen \& Markell, 1980). These classes are defined by whether or not observers perceive temporal brightness enhancement (the Broca-Sulzer effect) under two asynchrony conditions for pulses to be compared: simultaneous onset and simultaneous offset. Type $A$ observers perceive brightness enhancement for both asynchrony conditions; Type $B$ observers perceive brightness enhancement for simultaneous offset of pulses but not for simultaneous onset; Type $\mathbf{C}$ observers do not generate the BrocaSulzer effect under either asynchrony condition. Here we present supplementary measures on observers of all three types: (1) magnitude estimation of the brightness of single pulses of light of varying duration, (2) modulation sensitivity for sine-wave flicker, and (3) contrast sensitivity for moving sine-wave gratings. The magnitude estimation data differentiated the three types of observers, but flicker and motion sensitivity did not. The three classes of observers probably differ in the perceptual criteria they employ in judging the brightness of isolated pulses of light; they probably do not differ in their underlying neurophysiological responses.
\end{abstract}

Temporal brightness enhancement (the Broca-Sulzer effect) is a perceptual phenomenon in which a pulse of light of approximately 50-150 msec appears brighter than longer or shorter pulses. Bowen and Markell (1980) studied the occurrence of this phenomenon in a large sample $(n=80)$ of naive observers and found systematic individual differences. Observers compared the brightness of equal-luminance short and long pulses differing by a fixed duration (Bowen $\&$ Nissen, 1979; Bowen \& Pokorny, 1978). Temporal brightness enhancement was studied under two conditions of pulse asynchrony, simultaneous onset of the pulses to be compared or simultaneous offset.

Three distinct classes of observers could be identified. One class of observer (designated Type A and representing $57.5 \%$ of the sample) exhibited temporal brightness enhancement for both simultaneous onset

This research was supported by National Science Foundation Grant BNS 78-17779 to R. W. Bowen and by a grant from the United States Air Force Office of Scientific Research (AFOSR 800246) to R. Sekuler. The participation of Dr. Owsley was made possible by a grant from the National Institute of Aging (AG01251 ). We gratefully acknowledge the prompt and reliable service of the Chicago Transit Authority (CTA) in moving observers between laboratories. and simultaneous offset of the pulses to be compared. In other words, for both asynchrony conditions, at least one pulse duration could be found (in a range of $50-150 \mathrm{msec}$ ) that was judged brighter than shorter or longer pulses. Type B observers $(32.5 \%$ of the sample) perceived brightness enhancement for simultaneous offset conditions but not for simultaneous conditions, a result similar to that reported by Raab and Osman (1962). Type C observers (10\% of the sample) did not perceive brightness enhancement under either asynchrony condition.

In the present study, we first classified groups of observers as Type A, B, or C based on their brightness vs. duration functions. We then tested these observers on three other tasks requiring psychophysical responses to temporally modulated targets. The first required observers to estimate the magnitude of the brightness of single pulses of varying duration (Raab, 1962); the second assessed sensitivity to full-field sinusoidal flicker (Kelly, 1971); and the third measured contrast sensitivity for moving sine-wave gratings (Sekuler, 1975). These measures were intended to test hypotheses about the basis for the differences among classes of observers. In particular, Bowen and Markell (1980) proposed that either observers 
might adopt different perceptual criteria in making brightness judgments or observers might differ qualitatively in their neurophysiological responses to light pulses. We shall consider these alternatives, differences in perceptual criteria, and differences in neurophysiological responses, in turn.

By perceptual criteria, we mean the use that observers make of the sensory activity evoked by a brief pulse. This viewpoint assumes that differential responses in brightness discrimination experiments (or different psychophysical tasks, in general) reflect utilization by observers of different features of their sensory responses (Sternberg, 1979; Ventura, 1980; Wasserman \& Kong, 1979). An example may help to illustrate this position. Suppose that the sensory response to a brief flash were biphasic, with a positive lobe followed by a negative one, and the rectified peak of the response were greatest for pulses of intermediate duration (Kelly \& Savoie, 1978). If some observer functioned as a "peak detector," basing judgments on the local maximum of sensory activity, his data would resemble what Bowen and Markell obtained from Type A observers: some intermediate duration of flash would be judged brighter than shorter or longer flashes. If some other observer acted as an "integrator," basing judgments on the temporal integral of sensory activity, he would produce data like those Bowen and Markell obtained from their Type $C$ observers: a broad range of short pulses would be judged less bright than a longer pulse.

Admittedly, our example describes only one of many possible differential perceptual criteria. Other examples of this general class might also account for individual differences in brightness enhancement reported by Bowen and Markell (1980). The plausibility of such models does not depend critically on a particular temporal form for the sensory response to brief pulses.

A second position is that observers differ in their neurophysiological responses. By neurophysiological response, we mean the temporal waveform of the sensory activity evoked by a pulse of light. Again, an illustration may be helpful. Kelly and Savoie (1978) have shown that certain stimulus conditions (uniform 8-deg achromatic field) producing a bandpass flicker sensitivity function (modulation transfer function, MTF) yield a nonmonotonic pulse threshold intensity vs. duration function. This function resembles suprathreshold brightness enhancement. Other conditions (an 8-deg circular 4-c/deg grating target) generating a low-pass flicker function produced a monotonic intensity duration function. For the former conditions, the calculated impulse response function is multiphasic, with a pronounced negative lobe following an initial positive phase. For the latter conditions, the impulse response function is monophasic and positive.
Kelly and Savoie thus found that different stimulus conditions generate different impulse responses. But suppose, for the sake of argument, that, for a single set of stimulus conditions, one type of observer (like Type A) had a biphasic impulse response, while another observer (Type C) had a monophasic impulse response. Since an observer's temporal MTF and the impulse response function are Fourier pairs, if the impulse response functions of Type $A$ and Type C differed, their temporal MTFs would differ correspondingly. If Type A observers had biphasic impulse responses and Type $C$ observers had monophasic impulse responses, the MTFs of the two groups would be band-pass and low-pass, respectively. A band-pass temporal MTF would be associated with a nonmonotonic brightness duration relation (brightness enhancement), while a low-pass MTF would be associated with a monotonic brightness duration relation. ${ }^{1}$ These differences in sensory temporal waveforms are examples of differences in neurophysiological responses.

We designed a battery of psychophysical tasks to distinguish between perceptual criteria and neurophysiological responses as sources of individual differences in brightness perception. The battery consisted of (1) classification of observers by simultaneous brightness discrimination, (2) magnitude estimation of brightness for pulses of varying duration, (3) measurement of the temporal MTF, and (4) measurement of sensitivity to moving sinusoidal gratings. If observers' neurophysiological responses to pulses of light differed, we would expect psychophysical differences in magnitude estimates of pulse brightness, flicker MTFs, and, possibly, sensitivity to moving gratings. Several outcomes could be associated with differences in perceptual criteria. For example, the difference in perceptual criterion could be confined to judgments of pulse brightness alone. In that case, we would expect systematic individual differences in simultaneous brightness judgments and in magnitude estimates of pulse brightness, but not on the other two tasks. Alternatively, individual differences could be restricted to the simultaneous brightness discrimination task alone, diminishing the finding of Bowen and Markell (1980) to the status of a methodological oddity. Finally, differences in perceptual criteria could affect responses to any temporally modulated stimulus. This would lead to systematic individual differences on each task in our battery, an outcome that could also have been caused by general neurophysiological differences.

\section{METHOD}

\section{Observers}

Thirty undergraduates fulfilling a requirement of an introductory psychology course were tested. First, we determined to which class-Type A, B, or C-each observer belonged. Observers showing high variability on the initial brightness judg- 
ments were excluded from further participation in the experiment, and observers were screened for visual acuity of $6 / 6$ or better using a Bausch and Lomb orthorator. Observers were also screened for normal color vision, using the Dvorine Pseudo-Isochromatic plates. Of the observers remaining in the pool, 11 were selected to participate in the magnitude estimation, flicker sensitivity, and motion sensitivity experiments. All three Type $C$ observers in the pool participated in the full experiment; four Type $A$ and four Type B observers were selected at random.

\section{Procedure and Apparatus}

Classification of observers. Brightness vs. duration relations were measured using the following technique (Bowen \& Markell, 1980; Bowen \& Nissen, 1979; Bowen \& Pokorny, 1978). On each trial, the observer views two equal-luminance pulses of different durations, presented separately to the left and right eyes, and reports which pulse appeared brighter. The eye receiving the longer or shorter puise is varied randomly over trials. Resulting data relate the percentage of trials on which the longer pulse was judged brighter to the duration of the shorter pulse. The occurrence of temporal brightness enhancement is indicated by a dip in this function below $50 \%$ at intermediate pulse durations: the shorter pulse in a pair is consistently judged brighter than a longer pulse. Moreover, whether or not brightness enhancement is evident for a given condition, the function should be near $100 \%$ at short durations, where brightness is increasing with duration, and near $50 \%$ at long durations, where brightness is independent of duration.

The apparatus (Bowen \& Markell, 1980; Bowen \& Pokorny, 1978) generated a stimulus array consisting of two 34-min-diam circular targets presented separately to the right and left eyes and separated in haploscopic view by $2 \mathrm{deg} 50 \mathrm{~min}$. A circular fixation target, $13 \mathrm{~min}$ in diameter, centered between the targets, was binocularly fused by the observer. The test targets were achromatic; the light source for the apparatus was a 150-W tungsten halogen lamp (DZE/FDS). The pulsed targets had a luminance of $100 \mathrm{~cd} / \mathrm{m}^{2}$, and the fixation target had a luminance of $20 \mathrm{~cd} / \mathrm{m}^{2}$. The stimulus array was viewed in darkness at a distance of $.4 \mathrm{~m}$.

Eight shorter-pulse durations were tested: $10,32,50,63,80$, 100,200 , and $500 \mathrm{msec}$. The longer pulse for the relative brightness judgment had a duration equal to that of the shorter pulse plus $500 \mathrm{msec}$. Stimuli were presented in blocks of five trials at a given shorter pulse duration. The position of the shorter pulse (right vs. left) was varied randomly within each block. The five trial blocks were presented in an ascending order, beginning with a shorter pulse duration of $10 \mathrm{msec}$ and increasing to a shorter pulse duration of $500 \mathrm{msec}$, under one of two pulse asynchrony conditions: simultaneous onset of shorter and longer pulses or sinultaneous offset of the pulses. Each asynchrony condition was replicated four times, according to either an ABBA or BAAB pattern ( $A=$ simultaneous onset, $B=$ simultaneous offset). In total, for every asynchrony condition, each of the eight shortlong pulse comparisons was presented to the observer 20 times, 10 times with the shorter pulse on the left of the stimulus array and 10 times with the shorter pulse to the right.

An observer thus made a total of 320 relative brightness judgments in the course of a single 1-h experimental session.

Magnitude estimation of brightness. In a separate, subsequent 1-h session, each observer made magnitude estimates of the brightness of single pulses of light with a free modulus method (Engen, 1971). On a given trial, the observer was presented with a single pulse at either the left or the right of the stimulus array described above. Eleven stimulus durations were employed: all of those used for the shorter pulse in the brightness comparison procedure plus 130,160 , and $320 \mathrm{msec}$.

Ten blocks of 22 stimuli were presented. Within a block, the 11 stimulus durations and two positions (left vs. right) were randomized. Observers were instructed to assign a number reflecting the apparent brightness of each pulse, using whatever numbers seemed to them to be appropriate.
Both the brightness discriminations data and the magnitude estimation data were collected in the Vision Laboratory, Department of Psychology, Loyola University of Chicago. Observers subsequently traveled on the elevated train ("El"; see Carpenter, 1973) to the Cresap Neuroscience Laboratory, Northwestern University, to be tested for flicker and motion sensitivity.

Flicker sensitivity. The $4.5 \times 5.5 \mathrm{deg}$ cathode ray tube display (P-32 phosphor) had a mean luminance of $60 \mathrm{~cd} / \mathrm{m}^{2}$. It was surrounded by a large iso-luminance screen, $20 \times 20 \mathrm{deg}$. Steadied by a chin- and foreheadrest, the observer viewed the screen from a distance of $1.13 \mathrm{~m}$. Natural pupils were used, and observers wore their best corrections.

A computer controlled the flicker of a cathode ray tube, causing its luminance to vary sinusoidally in time. Whenever the temporal modulation was visible, the observer depressed a handheld switch and held it down. Sensing this, the computer decreased the amplitude of the temporal modulation by $4 \mathrm{~dB} / \mathrm{sec}$. Eventually, the amplitude of the modulation went below threshold. The observer then released the switch, causing the computer to increase the amplitude of modulation by $4 \mathrm{~dB} / \mathrm{sec}$. Depressing and releasing the switch, the observer caused the temporal modulation to effect a transition from invisibility to visibility and back again. In this way, the observer kept the grating's contrast around the threshold level.

The computer began with a flicker frequency of $1 \mathrm{~Hz}$, and the observer tracked his threshold for $30 \mathrm{sec}$. Then, after a pause of about $10 \mathrm{sec}$, the computer repeated the procedure at twice the modulation frequency. This procedure was repeated until testing was completed with $32 \mathrm{~Hz}$. The session ended with a repeat of the first frequency $(1 \mathrm{~Hz})$.

After one session's tracks were completed, the computer calculated the modulation amplitude for each track by averaging amplitudes at peaks and valleys of the track. The observer's sensitivity was defined by the reciprocal of the modulation threshold.

Contrast thresholds for moving gratings. A drifting sinusoidal grating $(1 \mathrm{c} / \mathrm{deg})$ was generated using standard techniques on an oscilloscope $63 \mathrm{~cm}$ from the observer's eye. The oscilloscope subtended $4 \times 4 \mathrm{deg}$ and had a mean luminance of $60 \mathrm{~cd} / \mathrm{m}^{2}$. Thresholds were determined by a method of limits. On alternate trials, thresholds were approached from randomly chosen subthreshold and suprathreshold points. The experimenter adjusted the contrast of the grating in steps of $1 \mathrm{~dB}$ until the observer indicated he saw something on the screen (approaching threshold from below) or until the observer indicated he could no longer see anything on the screen (approaching threshold from above). Two ascending and two descending series were run at each of two speeds of grating movement, .5 and $10 \mathrm{~Hz}$. Order of testing at the two movement speeds was randomized. The threshold at each speed of movement was the mean of the four estimates taken at that speed. Sensitivity at each movement speed was defined by the reciprocal of the contrast threshold.

\section{RESULTS}

\section{Classification of Observers}

Figure 1 shows the mean percentage of trials on which the longer pulse was judged brighter as a function of the duration of the shorter pulse for observers designated as Type $A(n=4)$, Type $B(n=4)$, and Type $C(n=3)$. Filled circles and solid lines indicate the simultaneous offset asynchrony condition; open circles and broken lines indicate the simultaneous onset condition. Error bars in Figure 1 show the $95 \%$ confidence intervals for the means.

We used the following criterion to classify observers (Bowen \& Markell, 1980). Temporal bright- 


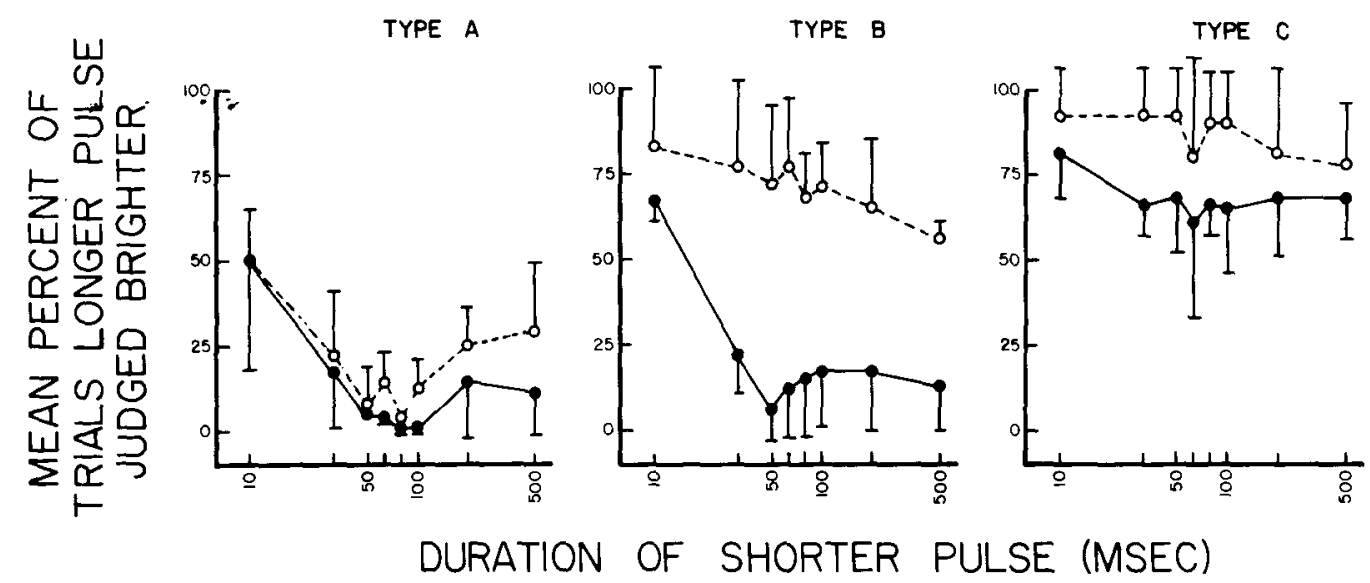

Figure 1. Plots of the percent of trials on which the longer pulse was judged brighter as a function of the duration of the shorter pulse. Data for observers classified as Type $A$ are given in the left-hand panel; data for Type B observers are given in the middle panel; and data for Type C observers are shown in the right-hand panel. See text for explanalion of classification criteria.

ness enhancement was deemed to occur for either asynchrony condition if at any shorter pulse duration the function was at $30 \%$ or less "longer pulse judged brighter," because 6 trials of 20 is significantly lower $(p<.057)$ than $50 \%$ for a binomial distribution with an assumed probability of responding "longer pulse brighter" of .5. For observers classified as Type A, then, temporal brightness enhancement occurs (indicated by local minima for both functions in the left-hand panel of Figurel) for both asynchrony conditions (filled circles); for Type B, enhancement is evident for the simultaneous offset condition, but not for simultaneous onset (open circles); and for Type C, enhancement does not occur under either asynchrony condition.

Our pool of 30 observers consisted of roughly $47 \%$ Type A, $43 \%$ Type $B$, and $10 \%$ of Type C.

\section{Magnitude Estimation of Brightness}

Figure 2 gives the geometric means of pulse brightness magnitude estimates as a function of pulse duration. The free modulus method we used permits dif- ferent observers to use different ranges of response numbers. These individual preferences for response numbers were unrelated to whether an observer was Type A, B, or C. But, to correct for differing preferences, data were normalized by the standard method (Engen, 1971). Error bars, calculated from the normalized data, represent $95 \%$ confidence intervals. The occurrence of temporal brightness enhancement in magnitude estimation data is indicated by a peak in the function at intermediate durations (Raab, 1962). Type A observers exhibit a pronounced peak at $63 \mathrm{msec}$, thus showing the Broca-Sulzer effect for judgments of the brightness of single pulses of light. The data of Type $\mathrm{C}$ observers indicate that brightness increases with increasing duration, without an obvious peak at any duration in the range we tested. Thus, observers who failed to show brightness enhancement with pairs of pulses also do not show enhancement with a single-stimulus method.

Type B observers show a brightness vs. duration relation intermediate in form between those for Type $A$ and Type $\mathrm{C}$ observers. There is some suggestion of

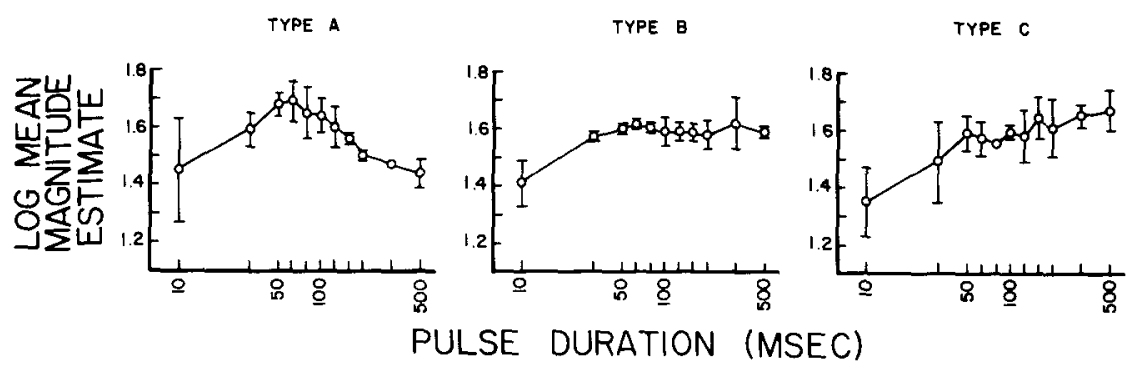

Figure 2. Log mean magnitude estimates of pulse brightness as a function of pulse duration (logarithmic scale) for the three classes of observers. 

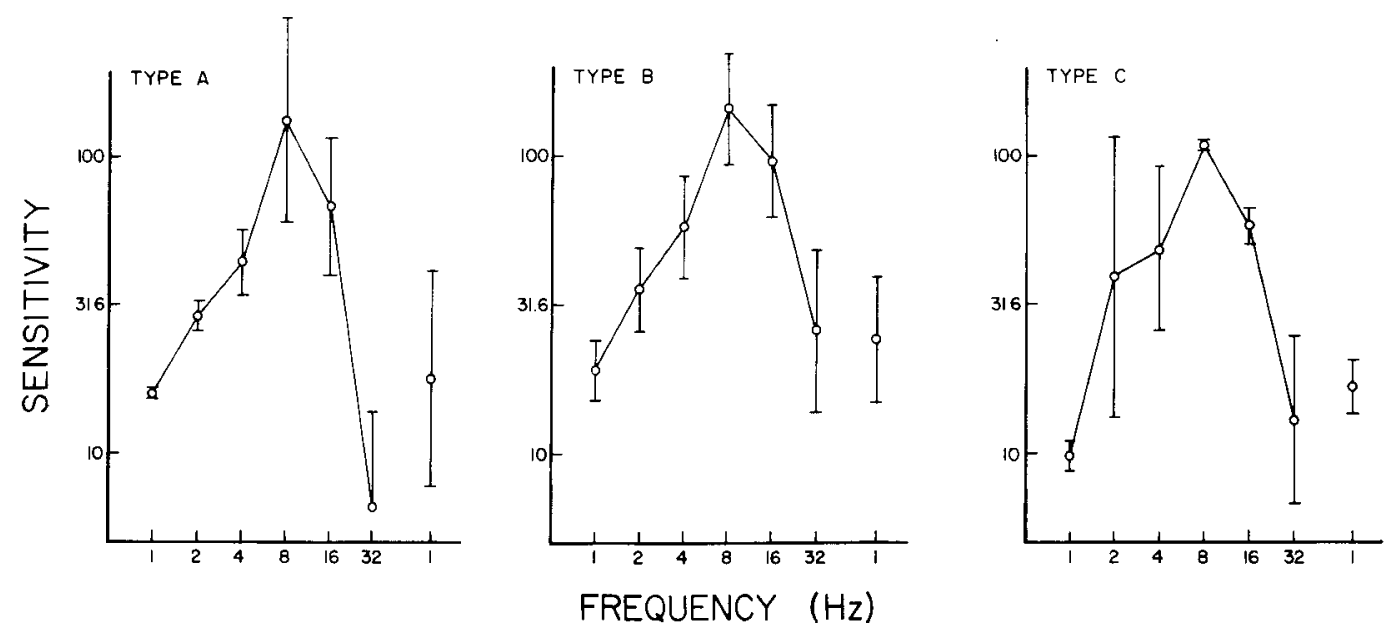

Figure 3. Mean modulation sensitivity (reciprocal of threshold contrast) to full-field flicker as a function of temporal frequency for the three classes of observers. The isolated point at the right of each panel is a replicated value for $1 \mathrm{~Hz}$ (see text).

a local peak in the function at $63 \mathrm{msec}$, but magnitude estimates of brightness are nearly constant from 100 to $500 \mathrm{msec}$ and do not decline sharply in this range, as do the data for Type A observers. Inspection of individual magnitude estimation functions indicates that two of the four Type B observers show a slightly peaked function, while the remaining two observers have functions that resemble the mean data for Type $\mathrm{C}$ observers.

In general, these results indicate that at least two classes of observers-Types A and C-can be clearly distinguished by their brightness judgments of single pulses of light. Therefore, the systematic differences among observers are not crucially dependent upon the simultaneous brightness discrimination procedure employed here and in our previous study (Bowen \& Markell, 1980).

\section{Flicker Sensitivity}

Figure 3 shows mean sensitivity to sinusoidal fullfield flicker as a function of the flicker's temporal frequency. Sensitivity is the reciprocal of the contrast threshold. The isolated point at the right of each function is the replication of the sensitivity measurement at $1 \mathrm{~Hz}$. The Type $\mathrm{A}$ and Type $\mathrm{C}$ functions are based on two observers each. ${ }^{2}$ Error bars show $95 \%$ confidence intervals about the means. For the observers tested, it is evident that there are no differences in sensitivity to full-field flicker. All three classes of observers manifest a characteristic band-pass flicker sensitivity function (Kelly, 1971) with a peak at $8 \mathrm{~Hz}$ and depressed sensitivity at low temporal frequencies. Also, the rate of decline in sensitivity from peak is similar at both high and low frequencies for all classes of observers. The three types of observers cannot be differentiated on the basis of flicker sensitivity.

\section{Contrast Thresholds for Moving Gratings}

Figure 4 shows sensitivity (reciprocal contrast sensitivity) for a $1-\mathrm{c} / \mathrm{deg}$ sine-wave grating presented at drift rates of 1.5 and $10 \mathrm{~Hz}$. The points represent means of data for individual observers of the three types. At the $.5-\mathrm{Hz}$ drift rate, there is extensive overlap among the three types in sensitivity measures. At a drift rate of $10 \mathrm{~Hz}$, the data for one Type B observer showed unrealistically high sensitivity. Generally, however, it is not possible to distinguish clearly among the three classes of observers on the basis of their motion sensitivity.

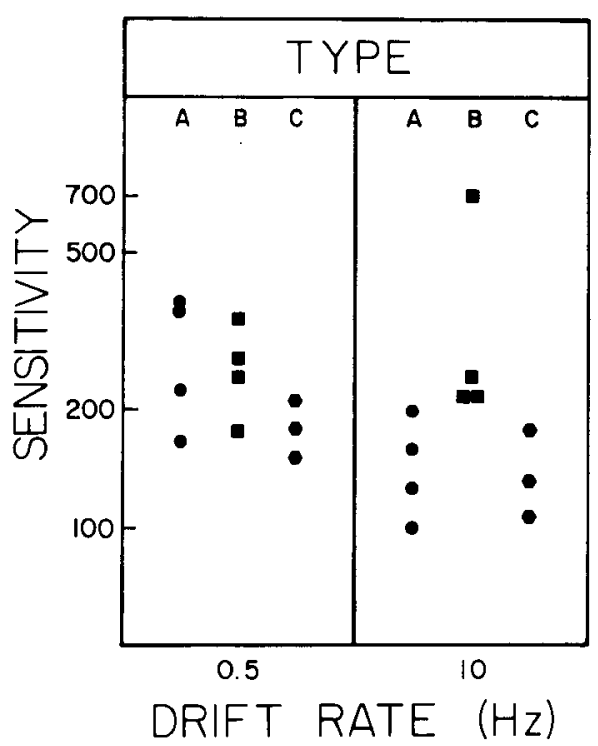

Figure 4. Motion sensitivity (reciprocal contrast threshold) for a 1-c/deg grating moving at .5 Hz (left panel) and at $10 \mathrm{~Hz}$ (right panel). Circles are from Type $A$ observers, squares are from Type B observers, and hexagons are from Type C observers. 


\section{DISCUSSION}

Our resultş show that systematic individual differences in pulse brightness perception are manifested in simultaneous brightness discrimination and magnitude estimation of pulse brightness, but not in measures of flicker and motion sensitivity. The homogeneous performance of all observer classes in the flicker and motion tasks rules out general differences in perceptual criteria and general differences in neurophysiological responses as bases for individual differences in brightness perception.

In this regard, it is important that Type A, B, and C observers all generate similar flicker functions. The characteristic depression of sensitivity at low temporal frequencies has been attributed to an inhibitory process (Kelly, 1971), and, as indicated above, the biphasic excitatory-inhibitory impulse response function derivable from the temporal modulation sensitivity curve (Kelly \& Savoie, 1978) implies a brightness enhancement effect for single pulses of light. Since Type C observers, who do not exhibit temporal brightness enhancement, nevertheless have normal temporal modulation sensitivity functions, the occurrence of the Broca-Sulzer effect must depend on factors beyond the basic aspects of visual temporal processing involving flicker sensitivity. Similarly, motion sensitivity did not differ significantly among observers of the three types. Type $A$ and Type C observers, who differ most substantially in their brightness enhancement functions, were virtually indistinguishable in their sensitivity to motion. Thus, whatever the origin of brightness enhancement, it clearly does not reflect some general mechanism governing all responses to temporal modulation.

There are different ways of distinguishing between those tasks that differentiated among classes of observers and those that failed to. For example, in our study, tasks that did differentiate among observers involved suprathreshold judgments; tasks that did not differentiate involved threshold judgments. Since the visual response would be nonlinear under suprathreshold conditions, but linear under threshold conditions, individual differences in perception may depend upon the evocation of nonlinear responses. This hypothesis clearly merits an appropriate experimental test.

We have confirmed the existence of systematic individual differences in pulse brightness discrimination and have shown that these differences also affect judgments of the brightness of a single light pulse. The present results leave us with the possibility that distinct classes of observers use different perceptual criteria in judging the brightness of isolated pulses of light.

One interpretation of Kelly and Savoie's data (1978), cited above, is that temporal brightness en- hancement is mediated by the magnitude of sensory activity at stimulus offset but not by the magnitude of sensory activity at stimulus onset. This interpretation might hold that our Type A observers base their judgments on sensory activity evoked by pulse offset, while Type $C$ observers base their judgments on activity evoked by pulse onset. Furthermore, Type B observers may shift between using the response at pulse onset and using it at pulse offset, depending upon the prevailing temporal asynchrony among pulses to be compared.

The hypothesis that observers differ in perceptual criteria makes us wonder about the developmental forces causing our three classes of observers to adopt different and characteristic ways of using the neurophysiological information generated by pulses of light. Also, we wonder whether these perceptual strategies might not be modifiable by experience or appropriate training. The possibility that observers differ in the way they utilize sensory information parallels Felsten and Wasserman's suggestion (1979) that an observer can base his sensory judgment on different features of his neural response, depending upon the psychophysical task confronting him.

The systematic differences in brightness perception that we find among observers may also have important practical consequences. For example, visual warning devices used in transportation systems can be designed specifically to capitalize on brightness enhancement effects (Boynton, 1967). But our results clearly show that such signal devices may be suboptimal for a large fraction of potential consumers. Consequently, designers of such systems may wish to explore the use of alternative temporal codes, for example, hybrid codes incorporating both long and short pulses of light.

\section{REFERENCES}

Bowen, R. W., \& MARkell, K. A. Temporal brightness enhancement studied with a large sample of observers: Evidence for individual differences in brightness perception. Perception a Psychophysics, 1980, 27, 465-476.

Bowen, R. W., \& Nissen, M. J. Luminance, not brightness, determines temporal brightness enhancement with chromatic stimuli. Journal of the Optical Society of America, 1979, 69, 581-584.

Bowen, R. W., \& Pokonny, J. Target edge sharpness and temporal brightness enhancement. Vision Research, 1978, 18, 1691-1695.

Boynton, R. M. Progress in physiological optics. Applied Optics, $1967,6,1283-1293$.

Carpenter, T. A. (Ed.). Chicago's rapid transit (Vol. 1): Rolling stock (Bulletin 113 of the Central Electric Rail Fans' Association). Chicago: Gregg-Moore Lithographers, 1973.

DE LANGE, H. Research into the nature of the human foveacortex systems with intermittent and modulated light. II. Phase shift in brightness and delay in color perception. Journal of the Optical Society of America, 1958, 48, 784-789.

Enoen, T. Psychophysics. II. Scaling methods. In J. W. Kling \& L. A. Riggs (Ed.), Woodworth \& Schlosberg's Experimental psychology. New York: Holt, Rinehart \& Winston, 1971. 
Felsten, G., \& Wasserman, G. S. The photoreceptor sensory code for pattern identification during visual masking. Sensory Processes, 1979, 3, 230-239.

KeLLY, D. H. Theory of flicker and transient responses: I. Uniform fields. Journal of the Optical Society of America, 1971, 61, 537-546.

KeLLY, D. H., \& SAvore, R. E. Theory of flicker and transient responses. III. An essential nonlinearity. Journal of the Optical Society of America, 1978, 68, 1481-1490.

Kelly, D. H., \& van Norren, D. Two-band model of heterochromatic flicker. Journal of the Optical Society of America, 1977, 67, 1081-1091.

RAAB, D. Magnitude estimation of the brightness of brief foveal stimuli. Science, 1962, 135, 42-44.

RAAB, D., \& Osman, R. Effect of temporal overlap on brightness matching of adjacent flashes. Journal of the Optical Society of America, 1962, 52, 1174-1178.

Sekuler, R. Motion perception. In E. C. Carterette \& M. P. Friedman (Eds.), Handbook of perception (Vol. 5). New York: Academic Press, 1975.

STERnBERG, S. Sensory variables and stages of human information processing. The Behavioral and Brain Sciences, 1979, 2, 282-283.

Ventura, J. Foveal metacontrast. I. Criterion content and practice effects. Journal of Experimental Psychology: Human Perception and Performance, 1980, 6, 473-485.

Wasserman, G. S. Brightness enhancement and opponent-colors theory. Vision Research, 1966, 6, 689-699.
Wasserman, G. S., \& Kong, K. L. Absolute timing of mental activities. The Behavioral and Brain Sciences, 1979. 2, 243-304.

\section{NOTES}

1. Other evidence links a biphasic impulse response with brightness enhancement. The MTF for purely chromatic flicker is lowpass not band-pass (de Lange, 1958; Kelly \& Van Norren, 1977), and brightness enhancement is absent for iso-luminance hue substitution stimuli (Bowen \& Nissen, 1979). Luminance increments of colored light, which are associated with a band-pass MTF and therefore a biphasic impulse response (Kelly \& Van Norren, 1977), do generate brightness enhancement (Bowen \& Nissen, 1979; Wasserman, 1966).

2. The data for two Type A observers were excluded because we judged that they may not have performed the task according to instructions: one observer exhibited abnormally high sensitivity to 8- and $16-\mathrm{Hz}$ flicker (the form of this observer's flicker sensitivity function was, however, similar to the mean data for Type A observers shown in Figure 3), and the other observer generated erratic data. The remaining Type $\mathrm{C}$ observer could not be scheduled for testing.

(Manuscript received April 16, 1981;

revision accepted for publication September 28, 1981.) 